\title{
Los efectos del Tratado de Libre Comercio con Estados Unidos y los precios del maíz colombiano
}

\author{
The Effects of the Free Trade Agreement with the \\ United States and the Prices of Colombian Corn
}

\author{
Os efeitos do Acordo de Livre Comércio com os \\ Estados Unidos e os preços do milho colombiano
}

Johanna Tróchez González * Marisol Valencia Cárdenas **

Juan Carlos Salazar ***

DOI: https://doi.org/10.19053/01203053.v37.n65.2018.5988

Fecha de recepción: 14 de abril de 2017

Fecha de aprobación: 5 de diciembre de 2017

\footnotetext{
* MSc en Ciencias Estadísticas Universidad Nacional de Colombia. Docente de la Facultad de Ciencia Básica. Instituto Tecnológico Metropolitano. Medellín, Colombia. Correo electrónico: jtrochezg@unal.edu.co (D) https:// orcid.org/0000-0002-2137-3143

** Ingeniera industrial. MSc en Estadística. PhD en Ingeniería-Industria y Organizaciones. Docente investigadora, Tecnológico de Antioquia, Medellín, Colombia. Correo electrónico: mvalencia@unal.edu.co (iD) https://orcid. org/0000-0003-3135-3012

***Phd Estadística. Profesor asociado Universidad Nacional de Colombia. Medellín, Colombia. Correo electrónico: jcsalasa@unal.edu.co (D) https://orcid.org/0000-0003-2286-3627
} 


\section{Resumen}

Desde la década de los 90, Colombia ha firmado diferentes acuerdos de libre comercio con diversos países y comunidades, a fin de liberar los aranceles de importación de varios productos agropecuarios sensibles a grandes volúmenes de importaciones, como es el caso del maíz, alimento básico de la canasta familiar, el cual ha presentado una disminución en la producción en los últimos veinte años. Este artículo propone una evaluación de los efectos del Tratado de Libre Comercio celebrado con Estados Unidos a través del modelo lineal mixto, teniendo como variable respuesta los precios semanales del maíz blanco y amarillo comercializado en las principales plazas de mercado colombianas, en un lapso de tiempo comprendido entre junio de 1996 hasta diciembre de 2016. Donde se encontró una disminución en los precios de comercialización en el tiempo, generando repercusiones sobre el ingreso del productor de maíz colombiano por tonelada producida.

Palabras clave: modelo matemático, producto agrícola, precio agrícola, comercio internacional.

Clasificación JEL: Q13, N50, C33, C12, F53 


\section{Abstract}

Since the 1990s, Colombia has negotiated various free trade agreements with different countries and communities, eliminating import tariffs on different agricultural products that are sensitive to large volumes of importations, such as corn, a staple food in the family basket, which has shown a decrease in production in the last 20 years. This article proposes an evaluation of the effects of the Free Trade Agreement signed with the United States through the linear mixed model, having as a response variable the weekly prices of white and yellow corn marketed in the main Colombian market places in a period of time between June 1996 and December 2016, where there was a decrease in market prices, with repercussions on the incomes of Colombian corn producers.

Keywords: mathematical model, agricultural product, agricultural price, international trade. 


\section{Resumo}

Desde os anos 90 Colômbia concordou vários acordos de livre comércio com diferentes países e comunidades, liberando as tarifas de importação de diferentes sensível a grandes volumes de importação de produtos agrícolas, como no caso do milho amarelo, o alimento básico na cesta, que apresentou uma diminuição na produção ao longo dos últimos 20 anos. Este artigo propõe uma avaliação dos efeitos do pacto de livre comércio com os Estados Unidos através do modelo linear misto, com a resposta preços semanais variáveis de milho amarelo vendidos nas principais praças do mercado colombiano, durante um período de tempo entre junho 1996 até 2016 Dezembro, que encontraram uma diminuição dos preços no mercado, gerando impacto sobre o produtor de milho colombiana.

Palavras-chave: modelo matemático, produto agrícola, preço agrícola, comércio internacional 


\section{INTRODUCCIÓN}

Anteriormente, el sector agropecuario colombiano se encontraba protegido por el sistema andino de franjas de precios, el cual estabilizaba el costo de importación de productos agrícolas caracterizados por presentar precios internacionales altamente volátiles (Reina \& Oviedo, 2011); pero, en la actualidad, las políticas de comercialización están sujetas a los 13 acuerdos de libre comercio celebrados por Colombia con diferentes países y comunidades (MinCIT, 2017), situación que implica que el país debe enfrentarse a mercados con amplias ventajas competitivas.

Dentro de los objetivos de los tratados de libre comercio se encuentran el fomento de la producción nacional a través de la competencia justa y el incremento de las oportunidades de inversión. Sin embargo, en estos acuerdos no todos los sectores económicos resultan beneficiados, debido principalmente a que las condiciones de producción entre los países miembros no siempre son equitativas; por ejemplo, el sector de cereales, específicamente el sector agrícola del maíz, enfrenta una fuerte competencia, producto de la entrada en vigencia del Tratado de Libre Comercio (TLC) con Estados Unidos, conocido como el mayor productor y exportador de maíz del mundo (Nadal \& Wise, 2005). Este acuerdo entró en vigencia el 15 de mayo del año 2012 (MinCIT, 2012a), fecha desde la cual Colombia ha importado 2.1 millones de toneladas de maíz cáscara amarillo y 136000 toneladas de maíz cáscara blanco, sin arancel y sin 
ningún control de bioseguridad, a través de un contingente que crece a una tasa del $5 \%$ anual, bajo la modalidad de asignación de primer llegado primer servido, hasta el año 2024 cuando se termina el programa de liberación arancelaria, en el que podrá entrar cualquier cantidad de maíz (MinCIT, 2012b).

Estados Unidos presenta una clara ventaja competitiva, ya que el productor americano cuenta con infraestructura, subsidios, tecnología de punta, maquinaria y modificación genética de las semillas, lo que, por ende, genera una mayor productividad (FENALCE, 2011). Por su parte, en Colombia la producción es rudimentaria, el Gobierno ha tratado de incentivar y ayudar al productor a través de líneas crédito, bonificaciones en la producción nacional y otros programas de acompañamiento al productor como el "Plan País Maíz" y alianzas con la industria (MinAgricultura, 2011). No obstante, no es suficiente para alcanzar un nivel elevado de competitividad, ya que este sector enfrenta otras problemáticas como el contrabando, en el que los competidores ingresan granos por debajo de los precios locales. Todo este contexto ocasiona pérdidas económicas al productor, al percibir menos ingresos por cosecha; solo aquellos productores con capacidad de secar y almacenar sus cosechas están menos expuestos a la volatilidad de los precios (Superintendencia de Industria y Comercio [SIC], 2010).

El caso colombiano es similar al que experimentó México en el año 1994, tras la entrada en vigencia del Tratado de Libre Comercio de América del Norte (TLCAN) con Estados Unidos y Canadá, en el que el Gobierno mexicano permitió importaciones por encima del cupo negociado y evadió el pago de aranceles valorados en 1766 millones de dólares, lo que causó pérdidas formidables al fisco de ese país (Comisión Económica para América Latina y el Caribe [CEPAL], 2013). Esto, sumado a los bajos precios del maíz en el orden global (Fiess \& Lederman, 2004), ocasionó un debilitamiento del sector, en el que se puso en riesgo el empleo de 2 millones de campesinos, lo que motivó la creación del programa PROCAMPO, en el que se protegió e incentivó al productor tradicional de maíz, para mitigar los efectos sobre la población campesina (CEPAL, 2005). En este tratado también se evidenciaron efectos ambientales sobre las plantaciones mexicanas de maíz, las cuales fueron contaminadas por las variedades genéticamente modificadas provenientes de Estados Unidos (Nadal \& Wise, 2005).

En Colombia, desde antes de la entrada en vigencia de este tratado de libre comercio, ha existido gran preocupación por los efectos en la economía agrícola de este sector, es así como diversos 
autores han tratado de plantear diversos escenarios, para determinar dichos efectos. Dutoit, Hernández y Cristóbal (2010) evaluaron las políticas de comercialización del maíz en los países latinoamericanos y evidenciaron que desde los años 90 existe una tendencia a la disminución de los aranceles de importación del maíz; además estudiaron la transmisión de precios, es decir, hasta qué punto movimientos internacionales de los precios del maíz se reflejan en la evolución de los precios nacionales de países como Panamá y Costa Rica, donde encontraron que la transmisión de precios es más fuerte en el mercado mayorista que en el del productor local.

Tonconi (2009) evaluó los efectos de este tratado en el sector agrícola "maíz colombiano" a través de un enfoque de equilibrio parcial de la economía, usando la información de 1966-2005, $\mathrm{y}$ encontró que los precios de comercialización de maíz nacional disminuyen al nivel de los precios internacionales, ocasionando un aumento en la demanda y una disminución de la producción local.

Núñez, Carvajal y Bautista (2013) realizan un análisis prospectivo acerca de las ventajas y desventajas del Tratado de Libre Comercio con Estados Unidos, donde se resalta que uno de los sectores más vulnerables ante la firma de este tratado es el sector agrícola del maíz.
Dadas estas condiciones de desventaja para el sector del maíz en Colombia y el ejemplo de apertura comercial en la economía mexicana, se plantea la siguiente pregunta de investigación: ¿El aumento de las importaciones de maíz provenientes de Estados Unidos ha tenido un efecto de disminución en los precios de comercialización de maíz cáscara, amarillo y blanco, en las diferentes plazas de mercado colombianas? Para dar respuesta a esta pregunta se hace una evaluación de impacto, a través del modelo lineal mixto, con la variable precios comercializados semanalmente por kilo al consumidor de maíz cáscara amarillo y blanco, en diferentes plazas de mercado, con un lapso de tiempo comprendido desde junio de 1996 hasta diciembre de 2016, para así determinar de manera cuantitativa los efectos en el precio, antes y después de la entrada en vigencia del Tratado de Libre Comercio con Estados Unidos.

De resultar una disminución sobre los precios comercializados, se generan efectos tanto para el productor como para el consumidor, si bien es beneficioso para el consumidor de maíz amarillo, al percibir el producto con menor cantidad de dinero, el productor - en cambio - se ve directamente afectado al percibir menos ingreso $y$, por ende, menos utilidad por las toneladas producidas, lo que ocasiona una reducción de la producción nacional y, por lo tanto, dependencia de 
la producción de maíz de otros países (González \& Ávila, 2014).

En el segundo capítulo de este artículo se examina el estado de la cuestión acerca del maíz en Colombia y se revisan las importaciones de maíz según el país de procedencia. Allí se refleja cómo las importaciones provenientes de Estados Unidos han tomado ventaja sobre otros países importadores. En el tercer capítulo se ilustra la metodología usada para la evaluación de impacto, y en el cuarto capítulo se ajusta el modelo de evaluación de efectos del TLC, donde se halla una reducción sobre los precios de comercialización del maíz amarillo y blanco.

\section{EL SECTOR DEL MAÍZ EN COLOMBIA}

Actualmente existe una alta demanda mundial de maíz para múltiples usos, entre los que se encuentran la producción de aceite, almidón, hidrocarburos; la generación de nuevas biotecnologías y la transformación genética. Sin embargo, en el mercado nacional, los principales usos son la alimentación de animales, la industria de alimentos balanceados y el consumo humano, con una demanda per cápita anual de maíz amarillo de 108.8 kilos por persona y de maíz blanco de 13.46 kilos por persona (Lample, 1995).
El cultivo de maíz es transitorio, lo cual significa que su ciclo vegetativo es relativamente corto, caracterizado por una alta rotación y un manejo eficiente de los factores de producción, tierra, mano de obra y capital. Este cultivo está ubicado principalmente en Córdoba, Sucre, Tolima y Valle del Cauca, $y$ es una fuente importante de generación de empleo en el sector rural, tanto directo como indirecto (FINAGRO, 2014), lo que lo ha convertido en uno de los cultivos más importantes en la producción nacional, ya que, según la Encuesta Nacional Agropecuaria, en los cultivos de ciclo corto el maíz amarillo ocupa mayor área con una participación del $27 \%$, seguido por la papa con una participación del $18 \%$ y el maíz blanco con una participación del $16 \%$ (Departamento Administrativo Nacional de Estadística [DANE], 2011). No obstante, los productores de maíz enfrentan serias dificultades a la hora de producir, debido a las plagas de los cultivos, los altos costos de los fertilizantes y las condiciones climáticas, pues las lluvias intensas afectan las cosechas.

Todos estos factores influyen para que el rendimiento de los cultivos de cereales en Colombia sea relativamente bajo en comparación con los países con los cuales Colombia ha firmado tratados de libre comercio, en especial con Estados Unidos, cuyos productores cuentan con subsidios, maquinaria y tecnología de punta que 
facilita las operaciones de siembra y cosecha. Esta situación se evidencia en la siguiente figura, donde se ilustran los rendimientos del cultivo de cereales desde el año 1961 hasta el año 2013, tiempo en el cual los rendimientos de Colombia siempre han sido inferiores en comparación con los rendimientos de Estados Unidos.

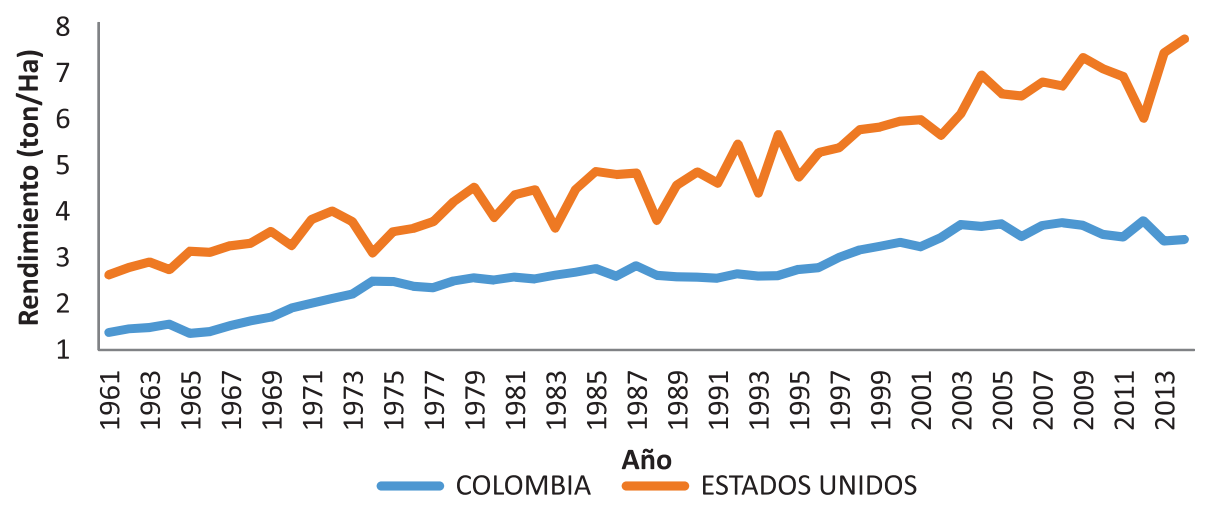

Figura 1. Comparación de rendimiento de cereales de Colombia con países asociados.

Fuente: elaboración propia con datos suministrados por el Banco Mundial (2017).

Es por ello que el Gobierno colombiano, por intermedio de la Federación Nacional de Cultivadores de Cereales y Leguminosas (FENAL$\mathrm{CE}$ ), ha incentivado la producción nacional de maíz, mediante créditos para la inversión en maquinaria, bonificaciones por tonelada cosechada y otros apoyos al productor colombiano, como el programa "Plan País Maíz" (FENALCE, 2010), el cual tenía como objetivo incrementar la seguridad alimentaria nacional, disminuir las necesidades de importación y aumentar la oferta de maíz amarillo tecnificado, lo que ocasionó un leve aumento en la producción, a través de los cultivos de maíz tecnificados (FENALCE, 2013).
Otra de las medidas del Estado colombiano fue la expedición de la Resolución 970 del año 2010 (Instituto Colombiano Agropecuario ICA, 2010), norma que regula la comercialización de semillas, las cuales deben ser protegidas, certificadas y registradas; hecho que ha generado gran polémica, debido a que la siembra de maíz se hace de manera tradicional, donde los pequeños productores, con extensiones de tierra menores a cinco hectáreas, almacenan sus mejores semillas de sus propias cosechas para siembras futuras (Confecampo, 2008). Por su parte, otros productores han cultivado con semillas certificadas y han encontrado que si bien se disminuye la necesidad de diferentes 
insumos agrícolas, dichas semillas no se han adaptado a las condiciones climatológicas locales, lo que ha ocasionado pérdidas económicas, producto de malas cosechas (SIC, 2010).

Todas estas dificultades en la producción y comercialización han generado una disminución en la oferta nacional de maíz, al pasar de autoabastecerse en la década de 1990, a depender actualmente de las importaciones de maíz (Vélez, 2002). La poca oferta de maíz cáscara amarillo, representada por la partida arancelaria 1005901100 , ha sido cubierta por otros países como
Argentina y Brasil, los cuales han abastecido el mercado colombiano, países caracterizados por ser los principales productores en Latinoamérica (United States Department of Agriculture [USDA], 2016). Pero, posteriormente, en el año 2012, tras la entrada en vigencia del TLC con Estados Unidos, se aumentaron las importaciones provenientes de este país (DANE, 2015), convirtiéndose en la actualidad en el principal exportador, como se observa en la siguiente figura, donde la línea vertical corresponde a la fecha de entrada en vigencia del Tratado de Libre Comercio con Estados Unidos:

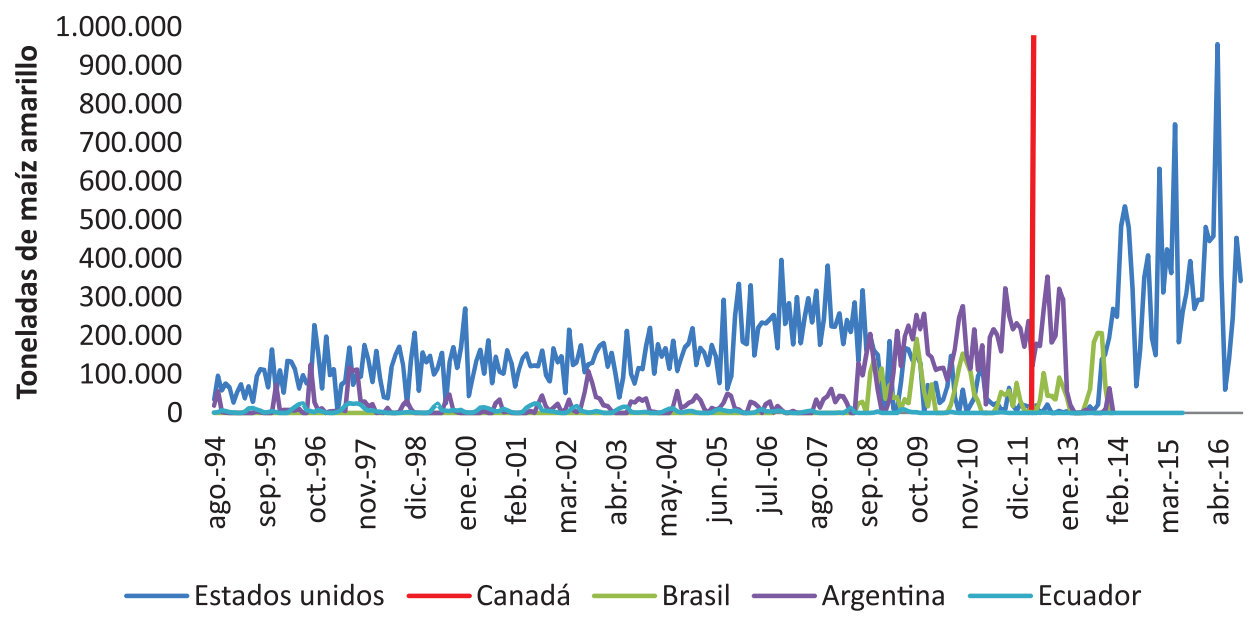

Figura 2. Importaciones en toneladas de maíz amarillo duro, partida arancelaria 1005901100.

Fuente: elaboración propia con datos del DANE (2015).

De la misma manera, el principal proveedor de maíz blanco en Colombia, representado por la partida arancelaria 1005901200, ha sido Estados Unidos.
Se observa en menor proporción maíz blanco proveniente de México, como se aprecia en la siguiente figura: 


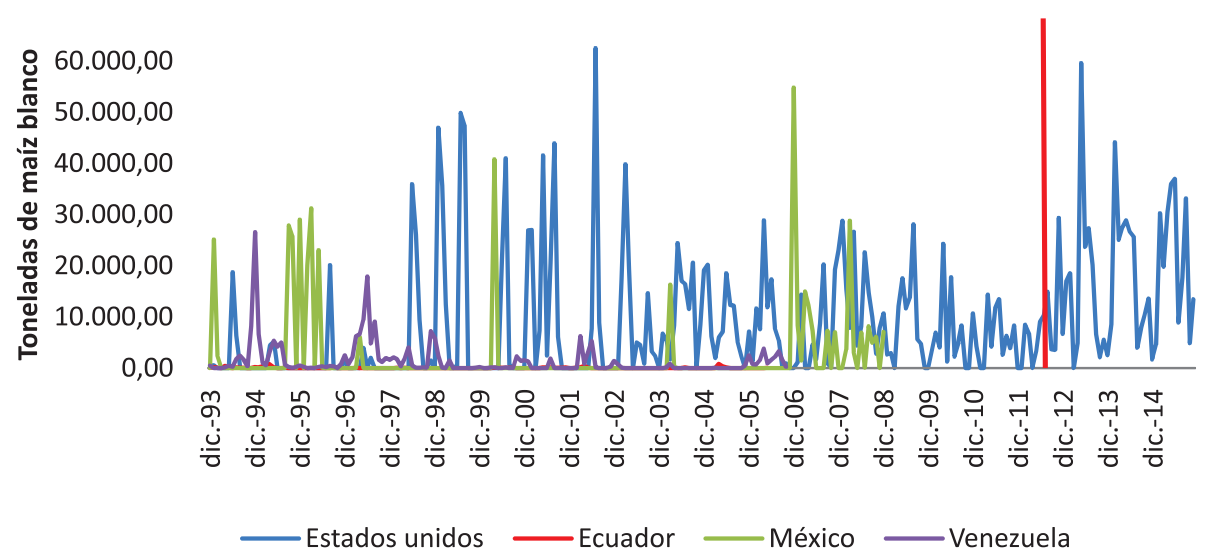

Figura 3. Importaciones en toneladas de maíz blanco duro, partida arancelaria 1005901200.

Fuente: elaboración propia con datos del DANE (2015).

Comparación de los precios corrientes de los diferentes tipos de maiz nacional y el maíz importado

La variable precios corrientes del maíz, tanto en el ámbito nacional como internacional, está expuesta a múltiples factores, uno de ellos es la variación de los precios del petróleo, la cual tiene repercusiones en los esquemas de producción y transporte, como el uso del maíz para la industria de hidrocarburos, por lo cual existe una amenaza de incremento de sus costos de venta en el mercado internacional (SIC, 2010).

Como se evidencia en las figuras anteriores, actualmente Estados Unidos es el principal exportador de maíz a Colombia, lo cual implica que todo cambio en volumen de producción, uso de nuevas tecnologías, efectos del cambio climático, costos, precios y cambios en los valores de la divisa, afectan en forma directa los niveles de abastecimiento nacional y por ende los precios de comercialización. Es así como en el año 2017, el precio de la divisa americana aumentó, lo que desestimuló en un $14 \%$, las importaciones de maíz proveniente de Estados Unidos a Colombia (Arango, 2017) y permitió comercializar a un buen precio la producción nacional de maíz.

En la siguiente figura se ilustran los precios de los diferentes tipos de maíz, nacionales e importados, desde abril del 2006 hasta octubre del 2015, donde es posible observar que ambos tipos de maíz tienen la misma tendencia en el tiempo, siendo superior el precio del maíz amarillo y blanco nacional, que el importado (FENALCE, 2011). 


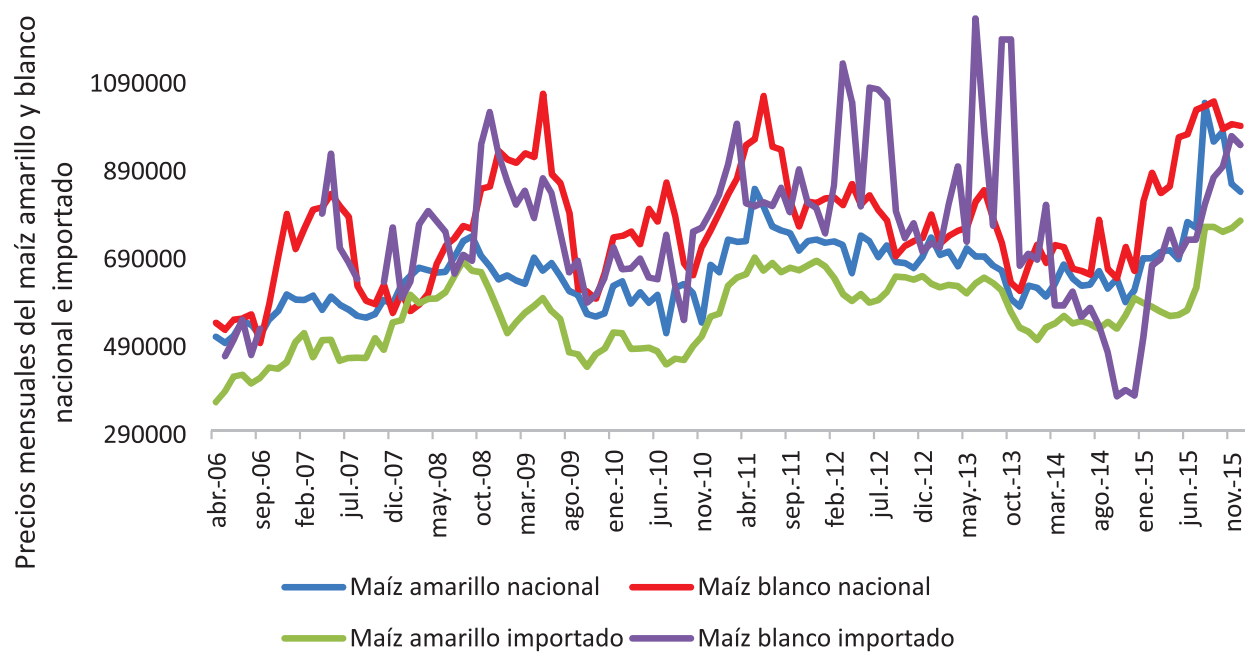

Figura 4. Precios mensuales del maíz amarillo y blanco nacional e importado.

Fuente: elaboración propia con datos de FENALCE (2016b).

\section{METODOLOGÍA}

El enfoque en la causalidad y la atribución es la característica distintiva de las evaluaciones de impacto, en su mayoría corresponden a modelos econométricos, a través del modelo contrafactual, el cual intenta responder a preguntas sobre causa y efecto, por medio del contraste de las respuestas en ausencia de la intervención, con las respuestas reales de la intervención (Gertler, Martínez \& Premand, 2011).
Para este caso interesa saber cuál ha sido el comportamiento sobre los precios de comercialización del maíz en diferentes plazas de mercados del país, antes y después de la entrada en vigencia del TLC, a través del modelo lineal mixto ajustado desde el software estadístico R Core Team (2015). Este modelo permite analizar la variabilidad entre sujetos y dentro de sujetos, a través de diferentes covariables en el tiempo, para así determinar el aporte a la variable respuesta (West, Welch \& Galecki, 2007). Está definido de la forma:

$$
\mathrm{y}_{i}=\mathrm{X}_{i} \beta+\mathrm{Z}_{i} b_{i}+\varepsilon_{i} \text { con } \mathrm{i}=1,2 \ldots \mathrm{n}
$$


Donde $\mathrm{X}_{i}$ representa los efectos fijos observados sobre los sujetos analizados, es decir, describen la relación entre la variable dependiente y las variables predictoras, lo que permite ver contrastes y diferencias entre los niveles de un factor fijo, mientras que los efectos aleatorios $Z_{i} b_{i}+\varepsilon_{i}$, representan características propias de cada sujeto (West et al., 2007).

Los vectores aleatorios en el $b_{i}$ vector siguen una distribución normal multivariada, $\mathrm{b}_{i}$ iid $N(0, D)$ donde

$$
D_{i}=\left[\begin{array}{cccc}
\operatorname{var}\left(b_{1 i}\right) & \operatorname{cov}\left(b_{1 i}, b_{2 i}\right) & \ldots & \operatorname{cov}\left(b_{1 i}, b_{2 i}\right) \\
\operatorname{cov}\left(b_{1 i}, b_{2 i}\right) & \operatorname{var}\left(b_{1 i}\right) & \ldots & \operatorname{cov}\left(b_{1 i}, b_{2 i}\right) \\
\vdots & \vdots & \ddots & \vdots \\
\operatorname{cov}\left(b_{1 i}, b_{2 i}\right) & \operatorname{cov}\left(b_{1 i}, b_{2 i}\right) & \ldots & \operatorname{var}\left(b_{1 i}\right)
\end{array}\right]
$$

Medida de bondad de ajuste $\mathbf{R}^{2}$ : es un valor que da cuenta de la proporción de varianza explicada por los efectos fijos y los efectos aleatorios estimados en el modelo lineal mixto, está definido como:

$$
R^{2}=\frac{\hat{\sigma}_{f}^{2}+\sum_{l=1}^{b} \hat{\sigma}_{f}^{2}}{\hat{\sigma}_{f}^{2}+\sum_{l=1}^{b} \hat{\sigma}_{l}^{2}+\hat{\sigma}_{\varepsilon}^{2}}
$$

Donde $\hat{\sigma}_{f}^{2}$ es la varianza de la componente de efectos fijos estimados, $\hat{\sigma}_{l}^{2}$ es la varianza debido a los b efectos aleatorios estimados para cada uno de los sujetos, $\hat{\sigma}_{\varepsilon}^{2}$ es la varianza del error aleatorio (Barton, 2016).

\section{Elección de las plazas de mercado}

La definición oficial, fijada por el Ministerio de Agricultura y Desarrollo Rural (MinAgricultura), en el artículo primero del Decreto 397 de 1995, establece que "se considera Mercado Mayorista aquella instalación o conjunto de locales construidos y adecuados para realizar actividades de intercambio comercial, por medio de la compraventa al por mayor de productos de origen agropecuario y pesquero, con el objeto de abastecer suficientemente a la población".

Teniendo en cuenta esta definición, se eligieron 19 plazas de mercado, de las principales ciudades del país (ver Tabla 1), las cuales garantizan el abastecimiento y distribución de alimentos en los centros urbanos. Ellas reflejan los precios corrientes de comercialización por kilo del maíz, a partir de la oferta y la demanda que llega a cada una de las plazas (FAO, 2010). 
Tabla 1. Principales plazas de mercado en Colombia

\begin{tabular}{|l|l|}
\hline \multicolumn{1}{|c|}{ Departamento } & \\
\hline Antioquia & $\begin{array}{l}\text { Central mayorista de Antioquia, con sede en Medellín } \\
\text { Plaza de mercado de Rionegro }\end{array}$ \\
\hline Cundinamarca & $\begin{array}{l}\text { Plaza de mercado de Paloquemao } \\
\text { Corporación de Abastos de Bogotá (Corabastos) }\end{array}$ \\
\hline Atlántico & $\begin{array}{l}\text { Plaza de mercado de Barranquilla (Granabastos) } \\
\text { Plaza de mercado de Barranquillita }\end{array}$ \\
\hline Santander & $\begin{array}{l}\text { Centro abastos en Bucaramanga } \\
\text { Cúcuta }\end{array}$ \\
\hline Cartagena & Bazurto \\
\hline Ibagué & Plaza El Jardín \\
\hline Manizales & Central Mayorista de Manizales \\
\hline Montería & Plaza mayorista de Montería \\
\hline Neiva & Central de abasto del sur \\
\hline Pasto & Plaza de mercado de Potrerillo \\
\hline Quindío & Plaza de mercado de Armenia (Mercar) \\
\hline Sincelejo & Plaza de mercado de Sincelejo \\
\hline Valledupar & Mercabastos \\
\hline Valle del Cauca & $\begin{array}{l}\text { Central Mayorista de Cali (CAVASA) } \\
\text { Plaza de mercado de Cartago }\end{array}$ \\
\hline
\end{tabular}

Fuente: FAO (2010)

\section{RESULTADOS}

Se analizaron los precios por kilo semanales del maíz amarillo en 19 plazas de mercado (Figura 5), y de maíz blanco en 11 plazas de mercado (Figura 6), disponibles en Agronet (2017), desde junio de 1996 hasta diciembre del 2016, para un total de 1047 precios por plaza en veinte años. Se observa que los precios tienen la misma tendencia en las diferentes plazas, algunas plazas no tienen todos los datos, sin embargo, el modelo lineal mixto se puede ajustar con datos faltantes. La línea vertical en ambas figuras indica la entrada en vigencia del Tratado de Libre Comercio con Estados Unidos, 15 de mayo de 2012. 


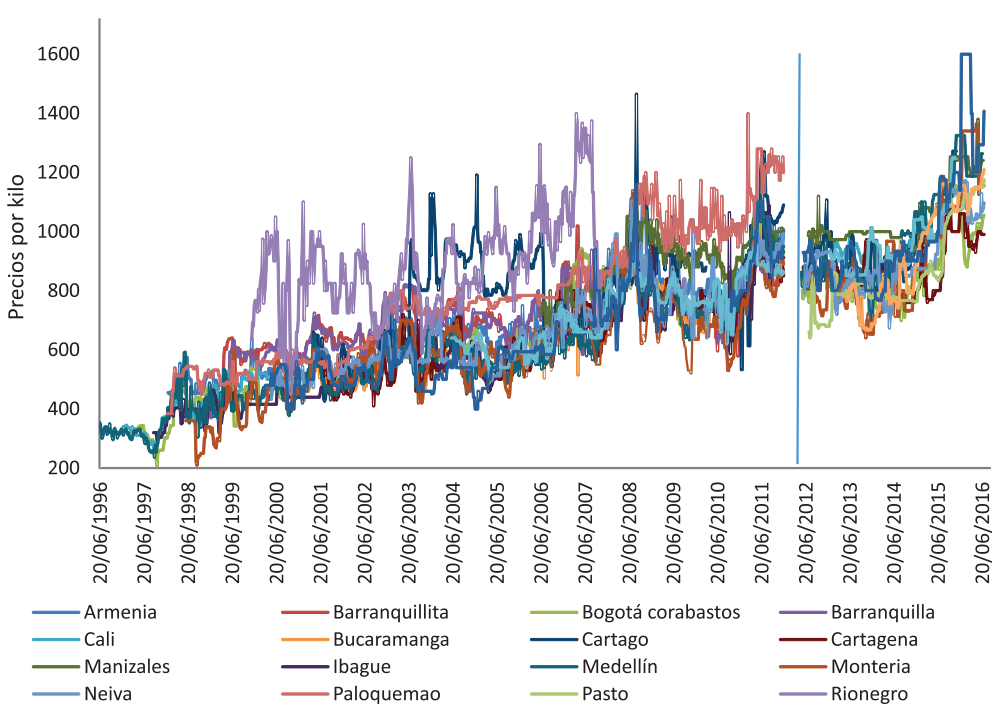

Figura 5. Precios semanales por kilo de maíz amarillo en 19 plazas del mercado colombiano.

Fuente: elaboración propia con datos de Agronet (2017).

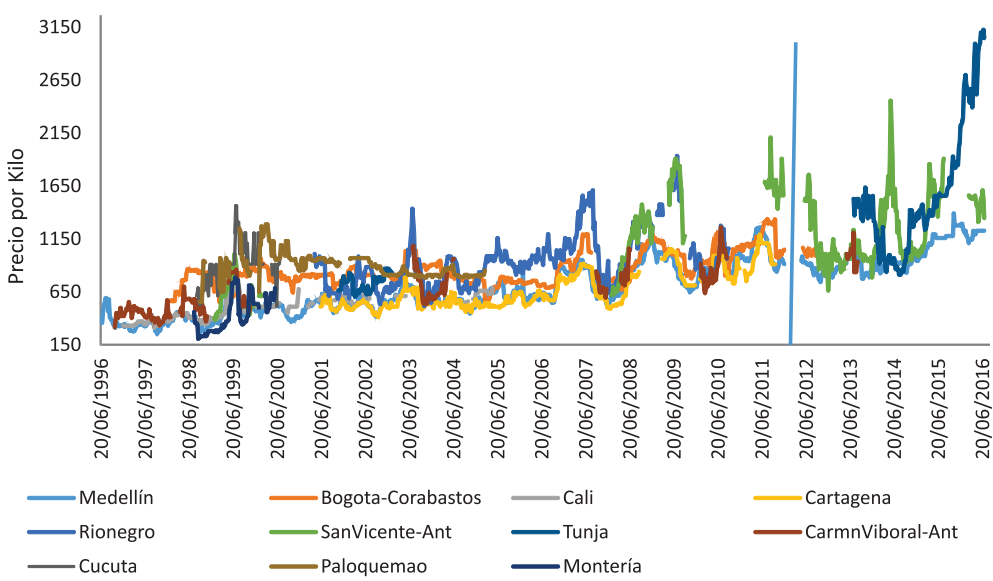

Figura 6. Precios semanales por kilo de maíz blanco en 11 plazas del mercado colombiano.

Fuente: elaboración propia con datos de Agronet (2017).

A partir de esta información, precios colombianas, se ajusta el modelo lipor kilo de maíz comercializados en neal mixto de intercepto y pendienlas principales plazas de mercado te aleatoria, en el que se asume la 
entrada en vigencia del TLC con Estados Unidos como una variable categórica, en donde se pretenden evaluar las siguientes hipótesis:

$H_{0}=$ no hay indicios de disminución de los precios de comercialización del maíz en las principales plazas de mercado colombiano.

$H_{1}=$ hay indicios de disminución de los precios de comercialización del maíz en las principales plazas de mercado colombiano.

Maíz amarillo: la siguiente tabla muestra la significancia de los parámetros estimados, donde se puede rechazar la hipótesis nula y concluir que la entrada en vigencia del TLC ha tenido efectos negativos sobre los precios de comercialización del maíz amarillo.

Tabla 2. Significancia de parámetros

\begin{tabular}{|c|c|c|c|c|c|}
\hline $\begin{array}{c}\text { Parámetro esti- } \\
\text { mado }\end{array}$ & Value & Std.Error & DF & t-value & p-value \\
\hline Intercepto & 158.7 & 141,46 & 11878 & 1.21 & 0.26 \\
\hline Tiempo & 0.902 & 0.1451 & 11878 & 6.21 & 0 \\
\hline Factor TLC & -64.77 & 3.87 & 11878 & -16.71 & 0 \\
\hline
\end{tabular}

La variable factor TLC genera dos modelos:

\section{Modelo antes del TLC}

$$
\mathrm{y}_{\mathrm{ij}}=158.7+0.902 t_{i j}+\mathrm{b}_{0 \mathrm{i}}+\mathrm{b}_{1 \mathrm{i}} t_{i j}+\varepsilon_{\mathrm{ij}}
$$

\section{Modelo después del TLC}

$$
\mathrm{y}_{\mathrm{ij}}=94.51+0.902 t_{i j}+\mathrm{b}_{0 \mathrm{i}}+\mathrm{b}_{1 \mathrm{i}} t_{i j}+\varepsilon_{\mathrm{ij}}
$$

La variable $\mathrm{y}_{\mathrm{ij}}$ representa el precio en la plaza $i$ con $i=1,2,3 \ldots, 19$, al tiempo $\mathrm{j}$ con $\mathrm{j}=1,2,3, . ., 1047$, la medida de bondad de ajuste $\mathrm{R}^{2}$ es de $95.03 \%$, que indica un buen ajuste en el modelo.
Donde se deduce que la entrada en vigencia del TLC tuvo un efecto de reducción en los precios del maíz amarillo de aproximadamente 65 pesos por kilo, lo que tiene repercusiones sobre el productor colombiano 
al percibir menos pago por cosecha, aproximadamente 65000 pesos por tonelada producida.

Maíz blanco: La siguiente tabla muestra la significancia de los parámetros estimados, donde se puede rechazar la hipótesis nula y concluir que la entrada en vigencia del TLC ha tenido efectos sobre los precios de comercialización del maíz blanco.

Tabla 3. Significancia de parámetros

\begin{tabular}{|c|c|c|c|c|c|}
\hline $\begin{array}{c}\text { Parámetro esti- } \\
\text { mado }\end{array}$ & Value & Std.Error & DF & t-value & p-value \\
\hline Intercepto & 408.39 & 90.94 & 4323 & 4.49 & 0 \\
\hline Tiempo & 1.07 & 0.3 & 4323 & 3.59 & 0 \\
\hline Factor TLC & -201.15 & 15 & 4323 & -13.41 & 0 \\
\hline
\end{tabular}

La variable categórica TLC genera dos modelos:

\section{Modelo antes del TLC}

$$
\mathrm{y}_{\mathrm{ij}}=408.39+1.07 t_{i j}+\mathrm{b}_{0 \mathrm{i}}+\mathrm{b}_{1 \mathrm{i}} t_{i j}+\varepsilon_{\mathrm{ij}}
$$

\section{Modelo después del TLC}

$$
\mathrm{y}_{\mathrm{ij}}=207.24+1.07 t_{i j}+\mathrm{b}_{0 \mathrm{i}}+\mathrm{b}_{1 \mathrm{i}} t_{i j}+\varepsilon_{\mathrm{ij}}
$$

La variable $y_{\mathrm{ij}}$ representa el precio en la plaza $i$ con $i=1,2,3 \ldots, 11$, al tiempo $\mathrm{j}$ con $\mathrm{j}=1,2,3, . ., 1047$, la medida de bondad de ajuste $\mathrm{R}^{2}$ es de $74.58 \%$, que indica un buen ajuste en el modelo.

Donde se deduce que la entrada en vigencia del TLC tuvo un efecto de reducción en los precios corrientes del maíz blanco de aproximadamente 200 pesos por kilo, lo que trae repercusiones sobre el productor colombiano al percibir menos pago por cosecha, aproximadamente 200000 pesos por tonelada producida.

Estos modelos y el estado del arte ofrecen evidencia para afirmar que la entrada en vigencia del TLC con Estados Unidos ha generado una disminución en los precios corrientes de comercialización en las diferentes plazas de mercado del país, lo que repercute en un desestímulo de la producción 
nacional, que a largo plazo representa problemas en el aspecto social, ya que el cultivo del maíz es uno de los principales generadores de empleo en el sector rural. Sin embargo, para las industrias que utilizan el maíz dentro de sus procesos productivos constituye una ventaja, pues al adquirir la materia prima a menores precios, logran, por ende, mayor utilidad.

\section{CONCLUSIONES}

Los reportes de la DIAN reflejan que a partir de la entrada en vigencia del tratado con Estados Unidos ha habido un aumento significativo en el volumen de maíz blanco y amarillo importado de este país, lo que ha ocasionado el desplazamiento de las importaciones de otros proveedores, tales como argentina y Brasil, principales productores de maíz en América Latina, situación que representa una dependencia de los precios nacionales del maíz de los precios del maíz de Estados Unidos.

La entrada en vigencia del TLC tuvo un efecto de reducción en los precios de comercialización del maíz en las diferentes plazas de mercado colombianas. Este valor es de aproximadamente 65 pesos por kilo en los precios de comercialización del maíz amarillo y 200 pesos por kilo en los precios de comercialización del maíz blanco, lo que trae repercusiones sobre el productor colombiano al percibir menos pago por cosecha, aproximadamente 65000 pesos y 200000 pesos por tonelada producida de maíz amarillo y blanco, respectivamente.

La disminución de los precios del maíz es beneficiosa para aquellas industrias alimenticias que usan este producto como insumo, al recibir la misma cantidad del producto por menor precio. Sin embargo, los productores son los afectados, ya que deben vender el maíz a los precios del maíz importado, percibiendo menos ganancia, e incluso sin obtener alguna ganancia, lo que ocasiona una desmotivación para la producción de maíz nacional.

El Estado colombiano debe continuar con políticas gubernamentales que fortalezcan e incentiven la productividad del sector, que promuevan el cooperativismo entre pequeños productores, y además debe establecer medidas de bioseguridad para revisar los alimentos que provienen de otros países, a fin de garantizar la preservación de la biodiversidad nacional.

Colombia debe fortalecer el sector durante la desgravación arancelaria para enfrentar la libre competencia en el año 2024, año en el que ingresarán libremente (sin ningún arancel) cantidades considerables de maíz a Colombia, provenientes de Estados Unidos.

\section{AGRADECIMIENTOS}

Los autores agradecen a Colciencias por su programa de Joven Investigador 2016 . 
Apuntes CENES Volumen 37, Número 65

enero - junio 2018. Págs. 151-172

\section{REFERENCIAS}

Agronet. (2017). Estadisticas. Recuperado de http://www.agronet.gov.co/agronetweb1/estadísticas.aspx

Arango, L. (2017, 24 de marzo). Sector agropecuario durante el 2016 y perspectivas para el 2017. Portafolio. Recuperado de http://www.portafolio. co/opinion/luis-arango-nieto/sector-agropecuario-durante-el-2016-y-perspectivas-para-el-2017-coyuntura-24-de-marzo-de-2017-504396

Banco Mundial. (2017). Rendimiento de los cereales. Recuperado de http://datos.bancomundial.org/indicador/AG.YLD.CREL.KG?end=2014\&star$\mathrm{t}=2014 \&$ view $=$ map

Barton, K. (2016). Multi model inference. MuMIn. Retrieved from https:// cran.r-project.org/web/packages/MuMIn/index.html

Comisión Económica para América Latina y el Caribe -CEPAL-. (2005). Efectos del TLCAN sobre las importaciones agropecuarias estadounidenses provenientes de México. Recuperado de https://www.cepal.org/es/publicaciones/4952-efecto-tlcan-importaciones-agropecuarias-estadounidenses-provenientes-mexico

Comisión Económica para América Latina y el Caribe -CEPAL-. (2013). Visión agrícola del TLC entre Colombia y Estados Unidos: preparación, negociación, implementación y aprovechamiento. Serie Estudios y Perspectivas, 25, 87. https://doi.org/http://hdl.handle.net/11362/4821

Departamento Administrativo Nacional de Estadística -DANE-. (2011). Encuesta nacional agropecuaria. Bogotá D.C.: DANE.

Departamento Administrativo Nacional de Estadística -DANE-. (2015). Importaciones colombianas. Recuperado de https://www.dane.gov.co/index.php/ comercio-exterior/importaciones

Dutoit, L., Hernández, K. \& Cristóbal, U. (2010). Transmisión de precios en los mercados del maiz y arroz en América latina. Statewide Agricultural Land Use Baseline 2015 (Vol. 1). Chile. https://doi.org/10.1017/ CBO9781107415324.004 
FAO. (2010). La función de los mercados mayoristas en los centros urbanos de Colombia, 32. Recuperado de http://coin.fao.org/coin-static/cms/media/6/12856000773900/funcion_mer_mayoristas_col.pdf

Federación Nacional de Cultivadores de Cereales y Leguminosas -FENALCE-. (2010). Fondo de Cereales Importados, informe de gestión. Bogotá D:C.: FENALCE.

Federación Nacional de Cultivadores de Cereales y Leguminosas -FENALCE-. (2011). El sector de cereales y leguminosas ante el TLC, (1). Bogotá D.C.: FENALCE.

Federación Nacional de Cultivadores de Cereales y Leguminosas -FENALCE-. (2016a). Indicadores económicos. Recuperado de http://www.fenalce.org/ nueva/index.php

Federación Nacional de Cultivadores de Cereales y Leguminosas -FENALCE-. (2016b). Indicadores cerealistas. Bogotá D.C.: FENALCE.

Fiess, N., \& Lederman, D. (2004). Mexican Corn: The Effects of NAFTA, 1-7. Recuperado de http://documents.worldbank.org/curated/en/2004/09/5754729/ mexican-corn-effects-nafta\%5Cnhttp://www-wds.worldbank.org/external/ default/WDSContentServer/WDSP/IB/2005/04/22/000090341_20050422 105517/Rendered/PDF/321090TradeNote18.pdf

FINAGRO. (2014). Perspectiva del sector agropecuario colombiano. Bogotá D.C.: FINAGRO.

Gertler, P. J., Martínez, S. \& Premand, P. (2011). La evaluación de impacto en la práctica. World Bank Group. DOI: 10.1596/978-0-8213-8541-8

Gonzalez, A. \& Ávila, J. (2014). El maíz en Estados Unidos y en México. Hegemonía en la producción de un cultivo. Argumentos, 75.

Instituto Colombiano Agropecuario -ICA-. (2010). Resolución 970 de 2010. https://doi.org/10.1017/CBO9781107415324.004

Lample, K. (1995, March). Rice Research: Food for 4 Billion People. GeoJournal, 35(3), 253-261. 
Ministerio de Agricultura -MinAgricultura-. (2011). Plan país maiz. En Cadenas productivas (p. 18). Bogotá D.C.: MinAgricultura.

Ministerio de Agricultura -MinAgricultura-. (1995). Reglamento de mercados mayoristas de productos agropecuarios. Recuperado de http://legal.legis. com.co/document?obra=legcol\&document=legcol_75992041479af034e$0430 \mathrm{a} 010151 \mathrm{f} 034$

Ministerio de Comercio, Industria y Turismo -MinCIT-. (2012a). Acuerdo de promoción comercial entre Colombia y Estados Unidos. Recuperado de http:// www.tlc.gov.co/publicaciones.php? $\mathrm{id}=727$

Ministerio de Comercio, Industria y Turismo -MinCIT-. (2012b). Apéndice I de Colombia_TLC_Capitulo 2., (c), 1-16. Recuperado de http://www.tlc.gov. co/loader.php?1Servicio=Documentos\&1Funcion=verPdf\&id=59269\&na$\mathrm{me}=02$ _Apendice_I_Colombia.final_letter.pdf\&prefijo=file

Ministerio de Comercio, Industria y Turismo -MinCIT-. (2013). Publicaciones. Recuperado de http://www.mipymes.gov.co/publicaciones.php?id=3635

Ministerio de Comercio, Industria y Turismo -MinCIT-. (2017). Retrieved from: http:/www.mincit.gov.co/tlc/publicaciones/7246/seguimiento_vision_nacional

Nadal, A., \& Wise, T. (2005). El comercio de maíz entre México y EE. UU. en el marco del NAFTA. En Globalización y medio ambiente: Lecciones de las Américas (pp. 49-92). EUA: Heinrich Böll Foundation North América.

Núñez, J., Carvajal, J. C. \& Bautista, L. A. (2013). El TLC con Estados Unidos y su impacto en el sector agropecuario colombiano: entre esperanzas e incertidumbres. Revista Electrónica de la Facultad de Derecho UDES, 1(1), 118-133. https://doi.org/10.1017/CBO9781107415324.004

Reina, M., \& Oviedo, S. (2011). Colombia y el TLC con la Unión Europea. Fescol Policy Paper, 4.

R Core Team. (2017). R: A Language and Enviroment for Statistical Computing. [Software]. R Foundation for Statistical Computing, Vienna, Austria. 
Superintendencia de Industria y Comercio -SIC-. (2010). Cadena productiva del maíz. Bogotá D.C.: SIC.

Tonconi, Q. (2009). Efectos del Tratado de Libre Comercio entre Colombia y Estados Unidos: un enfoque de equilibrio parcial para el sector agrícola maíz colombiano. Observatorio de la Economía Latinoamericana, 114. Recuperado de www.eumed.net/cursecon/ecolat/co/

United States Department of Agriculture -USDA-. (2016). Producción mundial de maiz 2016/2017. Recuperado de www.usda.gov

Vélez, G. (2002). Los cultivos y los alimentos transgénicos en Colombia. Grupo Semillas. Recuperado de http://www.semillas.org.co/es/los-alimentos-transgnicos-en-colombia

West, B., Welch, K. \& Galecki, A. (2007). Linear Mixed Models. A Practical Guide Using Statistical Software. USA: C. and Hall, Prentice-Hall. 